\title{
ALL REGULAR-SOLID VARIETIES OF IDEMPOTENT SEMIRINGS
}

\author{
Hippolyte Hounnon \\ Department of Mathematics \\ University of Abomey-calavi \\ Republic of Benin \\ e-mail: hi.hounnon@fast.uac.bj
}

\begin{abstract}
The lattice of all regular-solid varieties of semirings splits in two complete sublattices: the sublattice of all idempotent regular-solid varieties of semirings and the sublattice of all normal regular-solid varieties of semirings. In this paper, we discuss the idempotent part.
\end{abstract}

Keywords: semiring, hypersubstitution, regular hypersubstitution, regular hyperidentity, solid variety, regular-solid variety.

2010 Mathematics Subject Classification: 16Y60, 08B15, $08 \mathrm{C} 85$.

\section{REFERENCES}

[1] K. Denecke and H. Hounnon, All solid varieties of semirings, J. Algebra 248 (2002) 107-117. doi:10.1006/jabr.2002.9023

[2] K. Denecke and H. Hounnon, All pre-solid varieties of semirings, Demonstratio Math. XXXVII (2004) 13-34.

[3] K. Denecke and S.L. Wismath, Hyperidentities and Clones (Gordon and Breach Science Publishers, 2000). ISBN 9789056992354

[4] E. Graczyńska, On normal and regular identities, Algebra Universalis 27 (1990) 387-397. doi:10.1007/BF01190718

[5] U. Hebisch and H.J. Weinert, Semirings - Algebraic Theory and Applications in Computer Science (World Scientific, Singapore-New Jersey-London-HongKong, 1993).

[6] F. Pastijn, Idempotent distributive semirings II, Semigroup Forum 26 (1983) 151-161. doi:10.1007/BF02572828 
[7] R. McKenzie, G. McNulty and W.F. Taylor, Algebras, Lattices Varieties, Vol. 1 (Inc. Belmonts Califormia, 1987).

Received 13 December 2015 1st Revised 3 November 2016 2st Revised 11 January 2017 